\title{
Superspace Type II 4D Supergravity from Type II Superstring
}

\author{
Daniel Nedel ${ }^{* \dagger}$ \\ IFT/UNESP, São Paulo, Brazil \\ E-mail: daniel@ift.unesp.br
}

\begin{abstract}
We derive the equations of motion of type II $4 \mathrm{D}$ supergravity in superspace. This is achieved by coupling the Type II Berkovits' hybrid superstring to an $N=2$ curved background and requiring that the sigma-model has $\mathrm{N}=(2,2)$ superconformal invariance at one loop. We show that there are no anomalies in the fermionic OPE's and the complete set of compensator's equations is derived from the energy-momentum tensor. The equations of motion describe a hypertensorial and vectorial multiplet coupled to a $U(1) \times U(1) N=2$ Poincarè Supergravity.
\end{abstract}

Fourth International Winter Conference on Mathematical Methods in Physics 09 - 13 August 2004

Centro Brasileiro de Pesquisas Fisicas (CBPF/MCT), Rio de Janeiro, Brazil

\footnotetext{
${ }^{*}$ Speaker.

$\dagger$ The author is supported by a FAPESP post-doc fellowship. This research is part of the author PhD thesis, advised by Nathan Berkovits and supported by FAPESP.
} 
Superspace low-energy effective actions play an important role in the study of string theory. They provide important pieces of evidence for the existence of various dual descriptions of string theories and permit an off-shell description of the supergravity selected by string theory. Also, in the point of view of supergravity theories, the study of string corrections to the superspace equations of motion provides a new arena to develop futher superspace techniques, that can be very important to the development of supersymmetric theories [1].

One way to construct low-energy effective actions in string theory is looking for the lowenergy equations of motion. This is achieved by defining the sigma-model for the string in a curved background and requesting conformal invariance [2].

To derive the superspace equations of motion of the $\mathrm{N}=2$ supergravity theory that comes from Type II superstring, we need to formulate the sigma-model directly in terms of a target superspace and which can be quantized covariantly. This is not the case of sigma models built whith GreenSchwarz or RNS formalism. An alternative formalism for superstrings was discovered by Berkovits with local $\mathrm{N}=2$ worldsheet superconformal invariance.

This formalism is known as hybrid formalism and is related to the RNS formalism by a fieldredefinition [3]; it is especially well-suited for compactifications to four dimensions, where it allows manifestly $4 D$ super-Poincaré covariant quantization [3]. The coupling of the theory to background fields was discussed in [4]. In [7], the low-energy effective equations of motion of heterotic superstring were derived directly in superspace by means of this formalism. It was the first time that a kind of beta function calculation was made in a complete $4 D$ supersymmetric way.

In [5] Berkovits started the development of another formalism based on pure spinors; it allows manifestly $10 D$ super-Poincaré covariant quantization. In ref [6] the pure spinor's formalism was used to construct a sigma model directly in $10 D$ curved superspace and by means of a tree level analysis of this sigma model, the structure of $10 D$ superspace type II supergravity was derived. For the $4 D$ case, the hybrid formalism was explored in [8] and the complete set of type II torsion's constraints was derived from the hybrid type II sigma model. In addition, a field redefinition enabled to get information of the dilaton at tree level. It was shown that this information is consistent with the compensator's superspace type II equations of motion.

In this paper, a one loop analysis of the Type II hybrid sigma model is carried out and the superspace equations of motion for Type II supergravity are derived. The methods presented here are a generalization of the methods developed for the heterotic superstring in [7].

The core of the hybrid formalism lies in the fact that a critical $\mathrm{N}=1$ string can be formulated as a critical $\mathrm{N}=2$ string, without changing the physical content. This is achieved by twisting the ghost sector of the critical $\mathrm{N}=1$ string . After performing this embedding for the critical RNS superstring, a field redefinition allows the resulting $\mathrm{N}=2$ string to be made manifestly spacetime supersymmetric for compactifications to four dimensions. In this case, the critical $c=6$ matter sector splits into a $c=-3$ four-dimensional part and a $c=9$ compactification-dependent part. The action for this superstring can be written as : $S=S_{4 D}+\alpha^{\prime} S_{F T}+S_{c}$, where the first term describes the classical four dimensional part, the second one is the Fradkin-Tseytlin term which has the dilaton coupling and $S_{c}$ is the action for the compactification-dependent $c=9$ theory. This work does not concern about the fields that depend on compactification, so it is enough to concentrate just in the $N=(2,2) c=-3$ sector. The $\mathrm{N}=(2,2)$ superconformal currents are defined by the left and right energy-momentum tensors, $T$ and $\widehat{T}$; the left and right fermionic generators, $G, \bar{G}, \widehat{G}$ and 
$\widehat{\bar{G}}$; and the $U(1) \times U(1)$ currents, $J$ and $\widehat{J}$. They satisfy the algebra:

$$
\begin{aligned}
& T(z) T(w)=\frac{c / 2}{(z-w)^{4}}+\frac{2 T(w)}{(z-w)^{2}}+\frac{\partial T(w)}{(z-w)}, \\
& T(z) G(w)=\frac{\frac{3}{2} G(w)}{(z-w)^{2}}+\frac{\partial G(w)}{(z-w)}, \quad T(z) \bar{G}(w)=\frac{\frac{3}{2} \bar{G}(w)}{(z-w)^{2}}+\frac{\partial \bar{G}(w)}{(z-w)}, \\
& J(z) G(w)=\frac{G(w)}{(z-w)}, \quad J(z) \bar{G}(w)=\frac{\bar{G}(w)}{(z-w)}, \\
& J(z) J(w)=\frac{c / 3}{(z-w)^{2}}, \quad G(z) \bar{G}(w)=\frac{\frac{2}{3} c}{(z-w)^{3}}+\frac{2 J(w)}{(z-w)^{2}}+\frac{2 T(w)+\partial J(w)}{(z-w)} .
\end{aligned}
$$

The anti-holomorphic (or right-moving) generators satisfy the same algebra changing $(z, w)$ for $(\bar{z}, \bar{w})$. In order to write a closed string action in target curved space one needs the vertex operators for the massless fields. For the type II superstring described in the hybrid formalism there is no distinction between Ramond and Neveu-Schwarz vertex operators and both sectors are components of a superfield $U$ [4]. From the worldsheet point of view $U$ is an $N=(2,2)$ primary field of conformal weight zero and $U(1) \times U(1)$ charge zero.

The $\mathrm{N}=(2,2)$ primary field conditions and the gauge conditions imply that $U$ is a prepotential for an $\mathrm{N}=2$ conformal supergravity coupled to a hyper-tensorial multiplet. The gauge fields of supergravity sit in a Weyl multiplet with 24 bosonic and 24 fermionic off-shell components, while the matter fields are described by a hypertensorial multiplet with 8 bosonic and 8 fermionic offshell components. This prepotential represents the massless compactification-independent fields of the Type II superstring, without the dilaton. The dilaton does not couple classically in the action; it is part of the compensator fields and not part of the hypertensorial multiplet. To know the precise form of the off-shell $\mathrm{N}=2$ Poincarè supergravity that describes the low-energy effective action for Type II superstrings, one needs to know the compensators and the complete set of supergravity constraints, in particular the torsion constraints that break the conformal invariance. In general, for $N=2$ supergravity it is necessary two compensators to pass from conformal to Poincaré [10],[11]. From the $N=(2,2)$ worldsheet point of view the compensators are selected by the $2 D$ supersymmetry of the Fradkin-Tseytlin term, that requires the following ones: target chiral(anti-chiral) superfields $\phi_{c}\left(\bar{\phi}_{c}\right)$, that are related to a vector multiplet, and target twisted-chiral (anti-twisted-chiral) superfields $\phi_{t c}\left(\bar{\phi}_{t c}\right)$, that are related to a hypertensorial multiplet.

In an $\mathrm{N}=24 \mathrm{~d}$ curved background, the type II superstring action is written in terms of the vielbein $E_{M}{ }^{A}$. In the $N=(2,2)$ superconformal gauge the action is [4]:

$$
\begin{aligned}
S & =\frac{1}{\alpha^{\prime}} \int d^{2} z\left[\frac{1}{2} \Pi^{a} \bar{\Pi}_{a}+d_{\alpha} \bar{\Pi}^{\alpha_{+}}+d_{\dot{\alpha}} \bar{\Pi}^{\dot{\alpha}}+\widehat{d}_{\alpha} \Pi^{\alpha-}\right. \\
& +\widehat{d}_{\dot{\alpha}} \Pi^{\alpha_{+}}+\frac{1}{2} \bar{\Pi}^{A} \Pi^{B} B_{A B}+d_{\alpha} P^{\alpha \beta} \hat{d}_{\beta}+d_{\dot{\alpha}} P^{\dot{\alpha} \dot{\beta}} \hat{d}_{\dot{\beta}}+d_{\alpha} Q^{\alpha \dot{\beta}} \hat{d}_{\dot{\beta}}+d_{\dot{\alpha}} \bar{Q}^{\dot{\alpha} \beta} \hat{d}_{\beta} \\
& -\frac{\alpha^{\prime}}{2}\left(\bar{\partial} \rho+i \bar{\partial}\left(\phi_{c}-\bar{\phi}_{c}+\phi_{t c}-\bar{\phi}_{t c}\right)\right) \times\left(\partial \rho+i \partial\left(\phi_{c}-\bar{\phi}_{c}+\phi_{t c}-\bar{\phi}_{t c}\right)+a_{z}\right) \\
& \left.-\frac{\alpha^{\prime}}{2}\left(\partial \widehat{\rho}+i \partial\left(\phi_{c}-\bar{\phi}_{c}-\phi_{t c}+\bar{\phi}_{t c}\right)\right) \times\left(\bar{\partial} \widehat{\rho}+i \bar{\partial}\left(\phi_{c}-\bar{\phi}_{c}-\phi_{t c}+\bar{\phi}_{t c}\right)+\widehat{a}_{z}\right)\right],
\end{aligned}
$$


where $\Pi^{A}=\partial Z^{M} E_{M}{ }^{A}$ and $\bar{\Pi}^{A}=\bar{\partial} Z^{M} E_{M}{ }^{A}$. The coordinates of the $\mathrm{N}=2$ target tangent superspace are $Z^{A} \rightarrow\left(x^{a}, \theta^{\alpha_{+}}, \theta^{\dot{\alpha}_{-}}, \theta^{\alpha_{-}}, \theta^{\dot{\alpha}_{+}}\right)$and \pm is an $S U(2)$-index. $P^{\alpha \dot{\beta}}$ and $Q^{\alpha \dot{\beta}}$ are chiral and twisted-chiral field strengths of $\mathrm{N}=2$ conformal supergravity whose lowest components are the Type II RamondRamond field strengths. The left and right-moving fermionic fields are $d_{\alpha}$ and $\hat{d}_{\alpha}{ }^{1} ; \rho, \hat{\rho}$ are leftmoving (chiral) and right-moving (anti-chiral) bosons. The fields $a_{z}, \hat{a}_{z}$ are the worldsheet $U(1) \times$ $U(1)$ gauge fields ( $e^{i \rho}$ carries U(1) charge). Defining $\phi-\bar{\phi}=\phi_{c}-\bar{\phi}_{c}+\phi_{t c}-\bar{\phi}_{t c}$, the holomorphic generators are:

$$
\begin{aligned}
T & =\frac{1}{\alpha^{\prime}}\left[-\frac{1}{2} \Pi^{a} \Pi_{a}-d_{\alpha} \Pi^{\alpha_{+}}-d_{\dot{\alpha}} \Pi^{\dot{\alpha}_{-}}+\frac{\alpha^{\prime}}{2}[\partial(\rho+\phi-\bar{\phi})]^{2}\right]-\frac{1}{2} \partial^{2}\left(\phi_{c}+\bar{\phi}_{c}+\phi_{t c}+\bar{\phi}_{t c}\right), \\
J & =\partial(-i \rho+\phi-\bar{\phi}), \quad G=\frac{1}{i \alpha^{\prime} \sqrt{8 \alpha^{\prime}}} \exp (i \rho) d^{\alpha} d_{\alpha}-\partial\left(\frac{1}{i \sqrt{2 \alpha^{\prime}}} e^{i \rho} d^{\alpha} \nabla_{\alpha_{+}}\left(\phi_{c}+\phi_{t c}\right)\right), \\
\bar{G} & =\frac{1}{i \alpha^{\prime} \sqrt{8 \alpha^{\prime}}} \exp (-i \rho) d^{\dot{\alpha}} d_{\dot{\alpha}}-\partial\left(\frac{1}{i \sqrt{2 \alpha^{\prime}}} e^{-i \rho} d^{\dot{\alpha}} \nabla_{\dot{\alpha}_{-}}\left(\bar{\phi}_{c}+\bar{\phi}_{t c}\right)\right) .
\end{aligned}
$$

The anti-holomorphic ones are obtained from (3) by using hatted variables and replacing $\partial$ by $\bar{\partial}$ and $\phi_{c}$ by $\phi_{t c}$. The $N=(2,2)$ algebra derived in (1) for Type II superstring coupled to flat superspace must be satisfied in the curved sigma-model. However, in curved space one no longer has worldsheet fields satisfying free OPE's and a perturbative approach to check the $N=(2,2)$ algebra is necessary. As usual in string theory, $\alpha^{\prime}$ counts the number of loops in the two-dimensional quantum theory, but in the hybrid formalism the kinetic term of the bosons $\rho$ and $\hat{\rho}$ does not have an explicit factor of $\frac{1}{\alpha^{\prime}}$ in front of, and therefore the $\alpha^{\prime}$-perturbation theory does not make sense for these fields. This problem is solved by making the field redefinition: $\rho \rightarrow \rho-i\left(\phi_{c}-\bar{\phi}_{c}+\phi_{t c}-\bar{\phi}_{t c}\right)$ and $\widehat{\rho} \rightarrow \widehat{\rho}-i\left(\phi_{c}-\bar{\phi}_{c}-\phi_{t c}+\bar{\phi}_{t c}\right)$. After that, $\rho$ and $\hat{\rho}$ obey the same free field OPE's that we have used to derive the algebra $\mathrm{N}=2$ in (1); for the other fields perturbation theory will be used. Although this field redefinition permits a well defined $\alpha^{\prime}$ perturbation theory, the dependence of the fermionic currents on $\rho$ and $\hat{\rho}$ results in a new tree level dependence of the generators on $\phi_{c}$ and $\phi_{t c}$. Owing to this dependence the sigma model (2) is only consistent at tree level if the dilaton fields satisfy the following equations [8]:

$$
\begin{array}{ll}
\nabla_{\widehat{\gamma}}\left(\phi_{c}-\bar{\phi}_{c}+\phi_{t c}-\bar{\phi}_{t c}\right)=0, & \nabla_{a}\left(\phi_{c}-\bar{\phi}_{c}+\phi_{t c}-\bar{\phi}_{t c}\right)=0 \\
\nabla_{\widetilde{\gamma}}\left(\phi_{c}-\bar{\phi}_{c}-\phi_{t c}+\bar{\phi}_{t c}\right)=0, & \nabla_{a}\left(\phi_{c}-\bar{\phi}_{c}-\phi_{t c}+\bar{\phi}_{t c}\right)=0
\end{array}
$$

where was used: $T^{\widetilde{\alpha}}=\left(T^{\dot{\alpha}_{-}}, T^{\alpha_{+}}\right)$and $T^{\widehat{\alpha}}=\left(T^{\dot{\alpha}_{+}}, T^{\alpha_{-}}\right)$. Surprisingly, these equations are the Type II compensator's equations that are going to be derived here from one loop analysis. In (4) it was used the covariant derivative in the tangent superspace defined as:

$$
\nabla_{A}=E_{A}{ }^{M} \partial_{M}+\omega_{A \beta_{+}}{ }^{\gamma_{+}} M_{\gamma_{+}}{ }^{\beta_{+}}+\omega_{A \dot{\beta}_{-}}{ }^{\dot{\gamma}} M_{\dot{\gamma}_{-}}{ }^{\dot{\beta}}{ }_{-}+\omega_{A \beta_{-}}{ }^{\gamma_{-}} M_{\gamma_{-}}{ }^{\beta_{-}}+\omega_{A \dot{\beta}_{+}}{ }^{\dot{\gamma}_{+}} M_{\dot{\gamma}_{+}}{ }^{\dot{\beta}_{+}}+\Gamma_{A} Y+\widehat{\Gamma}_{A} \widehat{Y},
$$

where $\omega, \Gamma, \widehat{\Gamma}$ are the Lorentz and $U(1) \times U(1)$ connections, $M$ are the Lorentz generators and $Y, \widehat{Y}$ the $U(1) \times U(1)$ generators. It must be observed that there are two independent spacetime spinors in the Type II sigma-model, so one has two independent fermionic structure groups. Thus,

\footnotetext{
${ }^{1}$ Actually, they carry indices + and - , respectively, which is omitted.
} 
just like the two independent $\mathrm{U}(1)$ connections one has two independent sets of irreducible spin connections: $\omega_{A \alpha_{+}}{ }^{\beta_{+}}, \omega_{A \dot{\alpha}_{-}} \dot{\beta}_{-}$and $\omega_{A \alpha_{-}}{ }^{\beta_{-}}, \omega_{A \dot{\alpha}_{+}}{ }^{\dot{\beta}_{+}}$.The covariant derivative satisfies the algebra

$$
\left[\nabla_{C}, \nabla_{A}\right\}=T_{C A}^{B} \nabla_{B}+R_{C A E}{ }^{D} M_{D}^{E}+F_{C A} Y+\widehat{F}_{C A} \widehat{Y},
$$

where [ $\}$ means commutation (anti-commutation), $F$ and $\widehat{F}$ are the super $U(1) \times U(1)$ field strengths and $T, R$ are the supertorsions and supercurvatures; It was shown in [8] that the $\mathrm{N}=(2,2)$ structure of the hybrid formalism selects a different version of the $\mathrm{N}=2$ Poincare Supergravities described in [11]. For the type II superstring the $S U(2) / U(1)$ is fixed by the matter (hypermultiplet) and the compensators are dynamical (string gauge). Part of the hypermultiplet that is not fixed goes to the supergravity multiplet which, after imposing the conventional constraints, presents $32+32$ off-shell degrees of freedom. At the end we have an $N=2 U(1) \times U(1)$ Poincarè supergravity.

Next, we are going to analyse the sigma model at one loop. The strategy is the same as in [7]. A typical beta-function calculation does not guarantee the full $N=(2,2)$ superconformal invariance. The latter would only follow from a standard supersymmetric beta function calculation if the model could be formulated in $N=(2,2)$ superspace on the worldsheet, which does not seem possible. So, we need to check the $N=(2,2)$ algebra by calculating the OPE's perturbatively. At tree-level, there are no double contractions and it is necessary just to verify the part of the $\mathrm{N}=(2,2)$ algebra that depends on simple contractions. At one loop one has double contractions. However, as the part of the generators that comes from the Fradkin-Tseytlin term has already an $\alpha^{\prime}$ dependence, one needs just to evaluate tree level OPE's when these generators are involved. To perform the perturbative check of the OPE's (1) in a covariant way, we are going to use a background covariant expansion that preserves manifestly all the local symmetries of the target superspace. The traditional way to achieve such an expansion is to use a tangent vector that relates two points in target superspace, the classical field and the quantum fluctuations, then expand all the tensors in powers of this vector and use Riemann normal coordinates to covariantize the expansion [9]. Each tensor $T$ of the action is expanded as follows: $\Delta T=\left[y^{A} \nabla_{A}, T\right]$, where $y^{A}$ is the quantum flutuation. By applying iteratively the operator $\Delta$ we get a power series in $y^{A}$ and classical fields. For example:

$$
\Delta\left(\Pi^{B} \nabla_{B} y^{A}\right)=-Y^{D} \Pi^{B} y^{C} R_{C B D}{ }^{A}-\omega(A) y^{A} \Pi^{B} y^{C} F_{C B}-\widehat{\omega}(A) y^{A} \Pi^{B} y^{C} \widehat{F}_{C B} .
$$

Here $\Pi^{A}$ is a classical field and $\omega(A), \widehat{\omega}(A)$ are the $U(1) \times U(1)$ weights of the index A. The only ones different from zero are: $\omega\left(\alpha_{+}\right)=\hat{\omega}\left(\alpha_{+}\right)=\frac{1}{2}, \omega\left(\dot{\alpha}_{-}\right)=\hat{\omega}\left(\dot{\alpha}_{+}\right)=-\frac{1}{2}$. For the fermionic fields we choose the expansion: $d_{\alpha}=d_{\alpha}+D_{\alpha}$ ( $D_{\alpha}$ being a classical field) and the same for $\hat{d}_{\alpha}$. Now, we can describe the kind of calculation we intend to do. We need to expand the action up to order three in the quantum fields and two for the classical fields. The kinetic part of the expanded action provides the worldsheet propagators. In momentum space they are:

$$
\begin{aligned}
\left\langle y^{a}(p) y^{b}(l)\right\rangle & =\alpha^{\prime} \eta^{a b} \delta(p+l) \frac{1}{|p|^{2}} \quad\left\langle d_{\alpha}(p) y^{\beta+}(l)\right\rangle=\alpha^{\prime} \delta_{\alpha}^{\beta} \delta(p+l) \frac{-i p}{|p|^{2}} \\
\left\langle\widehat{d}_{\alpha}(p) y^{\beta-}(l)\right\rangle & =\alpha^{\prime} \delta_{\alpha}^{\beta} \delta(p+l) \frac{-i \bar{p}}{|p|^{2}},
\end{aligned}
$$

$\bar{p}$ being the complex conjugate of $p$. The other part of the action provides the vertices. By expanding the generators using the same background covariant expansion, the OPE's are calculated 
contracting the fields with the vertices in the action. By demanding that the $N=(2,2)$ algebra is satisfied at one loop, the equations of motion are derived for the background fields.

In order to avoid the problems with coordinate space regularizations, the OPE's are calculated in momentum space using the following dimensional regularization:

$$
\begin{aligned}
& \int d^{d} p \frac{p^{a} \bar{p}^{b}}{\left[|P|^{2}\right]^{\alpha}[|P-K|]^{\beta}}=k^{a+1-\alpha-\beta} \bar{k}^{b+1-\alpha-\beta}\left|\frac{K^{2}}{\mu^{2}}\right|^{-\varepsilon} \times \sum_{i=0}^{i=a}\left(\begin{array}{c}
a \\
i
\end{array}\right)\left[\frac{\Gamma(2-\alpha-\beta+b+i-\varepsilon)}{\Gamma(2-2 \varepsilon-\alpha-\beta+i+b)}\right. \\
& \left.\times \frac{\Gamma(\alpha+\beta-1-i+\varepsilon)}{\Gamma(1+\varepsilon) \mu^{-2 \varepsilon}} \Gamma(1-\varepsilon-\beta+i)\right] .
\end{aligned}
$$

After that we go back to coordinate space by means of an inverse Fourier transform. As we are calculating expectation values of conserved currents, all the divergences are canceled. The checking of all OPE's of the $N=(2,2)$ algebra is a tedious and hard work. Let's put just some results. For the $G(z) G(w)$ OPE there is no information at one loop. All the terms are proportional to the constraints derived in [8]. Also, we can show that there is no information coming from the $G(z) \widehat{G}(w)$ OPE. For $G(z) \bar{G}(w)$ the result is:

$$
\langle G(z) \bar{G}(w)\rangle=\frac{1}{(z-w)}\left[2 T(w)-\frac{1}{8 \alpha^{\prime}}\left[D^{\alpha} R_{\dot{\beta} \alpha}^{\dot{\beta} \dot{\gamma}} D_{\dot{\gamma}}+D^{\dot{\alpha}} R_{\beta \dot{\alpha}}^{\beta \gamma} D_{\gamma}-\frac{1}{2} D^{\alpha} D^{\dot{\gamma}} F_{\dot{\gamma} \alpha}\right]\right] .
$$

In this equation all the spinors have index $\alpha_{i}=\alpha_{+}$and $\dot{\alpha}_{j}=\dot{\alpha}_{-}$. The first term is the right term for this part of the $N=(2,2)$ algebra. The second one can be removed by redefining the expansion for $D^{\alpha}$. In general the anomalies that result in the equations of motion come from anti-holomorphic terms in the holomorphic OPE's and holomorphic terms in the anti-holomorphic OPE's. The result above shows that there is no information from fermionic currents. So, the equations of motion come from the OPE's that involve the energy-momentum tensor. The result for $T(z) G(w)$ is:

$$
\begin{aligned}
\langle T(z) G(w)\rangle & =\frac{(\bar{z}-\bar{w})}{(z-w)^{3}}\left[\bar{\Pi}^{d}\left(R_{d \beta_{+} \gamma_{+}}{ }^{\beta_{+}}+\frac{1}{2} F_{d \gamma_{+}}+\frac{1}{2} R_{\gamma_{+} b d}{ }^{b}-i T_{d \gamma_{+} \beta_{-}} \beta_{-}^{\prime}+2 \nabla_{d} \nabla_{\gamma_{+}}(\phi-\bar{\phi})\right) D^{\gamma_{+}}\right. \\
& \left.+\bar{\Pi}^{\widehat{\delta}}\left(R_{\widehat{\delta} \beta_{+} \gamma_{+}}{ }^{\beta_{+}}+\frac{1}{2} F_{\widehat{\delta} \gamma_{+}}+2 \nabla_{\widehat{\delta}} \nabla_{\gamma_{+}}(\phi-\bar{\phi})+i T_{\widehat{\delta} \widehat{\beta}, \gamma_{+}} \widehat{\widehat{\beta}}\right) D^{\gamma_{+}}+\ldots\right]+\frac{3 G}{2(z-w)^{2}} .(10)
\end{aligned}
$$

Here the dots mean terms that are higher derivatives of the first one. Now one has anti-holomorphic terms. These are the anomalies and provide the equations of motion. The holomorphic term is the right term for the $N=(2,2)$ OPE's and we got undesirable holomorphic terms that were cancelled redefining the energy-momentum tensor by means of contra-terms. Also it was necessary to redefine the $D$ expansion. The right expansion for the $D$ field is related to a shift symmetry in the background expansion and may be interesting to show that the selected expansion comes from Slavnov-Taylor identities. The results from $T(z) T(w)$ are not presented because it will take many pages and all the information about the equations of motion can be read from (10). The derived equations are:

$$
\begin{aligned}
R_{\widehat{\delta} \beta_{+} \alpha_{+}}{ }^{\beta_{+}}+\frac{1}{2} F_{\widehat{\delta} \alpha_{+}}+i T_{\widehat{\delta} \widehat{\beta}, \alpha_{+}}{ }^{\widehat{\beta}} & =-2 \nabla_{\widehat{\delta}} \nabla_{\alpha_{+}}(\phi-\bar{\phi}) \\
R_{d \beta_{+} \gamma_{+}}{ }^{\beta_{+}}+\frac{1}{2} F_{d \gamma_{+}}+\frac{1}{2} R_{\gamma_{+} b d}{ }^{b}-i T_{d \gamma_{+} \beta_{-}} \dot{\beta}_{-} & =2 \nabla_{d} \nabla_{\gamma_{+}}(\phi-\bar{\phi}) .
\end{aligned}
$$


From Bianchi identities we have (with constraints derived in [8]) :

$$
R_{\widehat{\delta} \beta_{+} \alpha_{+}}{ }^{\beta_{+}}+i T_{\widehat{\delta} \widehat{\beta}, \alpha_{+}}{ }^{\widehat{\beta}}=-\frac{5}{2} F_{\widehat{\delta} \alpha_{+}} .
$$

Using the fact that the combination: $\exp \left(\phi_{c}-\bar{\phi}_{c}+\phi_{t c}-\bar{\phi}_{t c}\right)$ has unitary $U(1)$ charge with respect to generator $Y$, the first equation can be written as:

$$
\nabla_{\alpha_{+}} \nabla_{\widehat{\delta}}\left(\phi_{c}-\bar{\phi}_{c}+\phi_{t c}-\bar{\phi}_{t c}\right)=0 \text {. }
$$

Making the same manipulations in the other equation one gets:

$$
\nabla_{\alpha} \nabla_{b}\left(\phi_{c}-\bar{\phi}_{c}+\phi_{t c}-\bar{\phi}_{t c}\right)=0
$$

The equations above are a derivative of the first line in (4).The same derivative of the second line is obtained from $\widehat{T} \widehat{G}$ OPE. Although the derivatives of an equation are a stronger condition than the equation itself, there is no physical meaning to take these stronger conditions into account. So the right equations of motion for the type II compensators derived from the sigma model at one loop are the equations (4). These equations of motion describe the $16+16$ degrees of freedom of a vector and a hypertensorial multiplet coupled to $\mathrm{N}=2$ supergravity in the string gauge. It will be interesting to take into account the compactification-dependent vertex operators. This extension is straightforward and it will be possible to see how the compactification will modify the supergeometry and the dynamics. The fact that there are no anomalies in the fermionic OPE's means that a traditional beta function calculation could provide the same equations of motion. There is no physical explanation for this fact, but it will be interesting to check this point in a two loop analysis.

\section{Acknowledgments:}

I would like to thank N. Berkovits, J. A. Helayel Neto, D. Z. Marchioro and M.C.B. Abdalla for useful discussions.

\section{References}

[1] S. J. J. Gates, A. Kiss and W. Merrell, Dynamical equations from a first-order perturbative superspace formulation, hep-th/0409104.

[2] E. Fradkin and A. Tseytlin, Quantum String Theory Effective Action, Phys. Lett. B 158 (1985) 316;

[3] N. Berkovits, The Ten-dimensional Green-Schwarz superstring is a twisted Neveu-Schwarz-Ramond string, Nucl. Phys. B 420 (1994) 332 [hep-th/9308129]; N. Berkovits and C. Vafa, On the Uniqueness of string theory, Mod. Phys. Lett. A 9 (1994) 653 [hep-th/9310170].

[4] N. Berkovits and W. Siegel, Superspace Effective Actions for 4D Compactifications of Heterotic and Type II Superstrings, Nucl. Phys. B 462 (1996) 213 [hep-th/9510106].

[5] N. Berkovits, Super-Poincare covariant quantization of the superstring, JHEP 0004 (2000) 018 [hep-th/0001035].

[6] N. Berkovits and P. S. Howe, Ten-dimensional supergravity constraints from the pure spinor formalism for the superstring, Nucl. Phys. B 635 (2002) 75. 
[7] J. de Boer and K. Skenderis, Covariant computation of the low energy effective action of the heterotic superstring, Nucl. Phys. B 481 (1996) 129 [hep-th/9608078].

[8] Daniel. L. Nedel, Consistency of superspace low energy equations of motion of 4D Type II superstring with Type II sigma model at tree-level, Phys. Lett. B 573 (2003) 217 [hep-th/0306166].

[9] L. Alvarez-Gaumé, D. Freedman and A. Mukhi, The Background Field Method And The Ultraviolet Structure Of The Supersymmetric Nonlinear Sigma Model, Annals of Physics 134 (1981) 85.

[10] S.J. Gates, Jr., Supercovariant Derivatives, Super Weyl Groups, And N=2 Supergravity, Nucl. Phys. B 176 (1980) 397; S. J. Gates, Jr., W. Siegel, Linearized N=2 Superfield Supergravity, Nucl. Phys. B 195 (1982) 39.

[11] B. de Wit and J. W. Van Holten, Structure Of N=2 Supergravity, Nucl. Phys. B 184 (1981) 77. 